\title{
A Neuro-fuzzy Logic Model Application for Predicting the Result of a Football Match
}

\author{
Uzochukwu C. Onwuachu and Promise Enyindah
}

\begin{abstract}
Many various models have been proposed with the goal of estimating the factors that determine the winner and losers in a football match, and many other models have been proposed with the goal of estimating the elements that determine the winner and losers in a football match. Predicting the result of a football match has been the interest of many gamblers and football fans all over the world. In this research, a Neuro-fuzzy logic model for forecasting the outcome of a football match is proposed. The suggested model comprises two phases: the first utilizes a neural network model to generate the primary factors that impact team performance; the second phase uses a neural network model to generate the major factors that affect team performance. In the second phase, a fuzzy logic model is used to forecast the outcome of a football match. MatLab 2008 was used to simulate the proposed system. In order to forecast the winner and loser of each football match, the model took into account a variety of parameters that affect both the host team and the visiting squad. The results show that the Neurofuzzy logic technique is an effective tool for forecasting the outcome of a football match.
\end{abstract}

Keywords - Fuzzy Logic, Football and Prediction, Neural Networks.

\section{INTRODUCTION}

Predicting the winner and the loser in a football match is a skill which needs to be developed with proper analysis of all the factors that can affect a particular match. Football these days has become one of the most popular sports attracting maximum number of fans all over the world. It is now an interesting research problem due to the difficulty in result prediction, because the result of a football match is dependent on many factors, such as a team's morale, skills, current score, etc. So even for football experts, it is very hard to predict the exact results of football matches [1]. However, many algorithms have been used for this prediction and they have their various challenges. These challenges are often induced due to emotion or lack of adequate datasets. Hence, in this recent time data mining techniques have attracted so many attentions [8]. The performance prediction is related to many people, including the football coach, football fans and the players. It also raises interest of researchers [5], in other to solve the prediction problem we explore the use of advanced non-linear modeling techniques such as neural networks and fuzzy logic model [12] to provide good analysis in such decision making. This paper proposes a Neuro-fuzzy logic model for predicting the result of a football match. The proposed model has two phases; the first phase uses a neural

Submitted on January 07, 2022.

Published on February 16, 2022.

Uzochukwu C. Onwuachu, Imo Sate University, Owerri, Imo State, Nigeria.

(e-mail: uzochukwuchristian@yahoo.com) network model to generate the major factors that affect team performance. The second phase's uses fuzzy logic model to predict the result of a football match. The model considered different factors that affects for both the host team and the guest in order to predict the result of each football match most especially using their previous past result.

\section{LITERATURE REVIEW}

Alexander et al. [2] worked on prediction of the results of football games based on fuzzy model with genetic and neurotuning. In their research, football game result prediction uses information from previous results of participating teams. The results generated from their simulation were achieved using fuzzy rules tuning on the data from league tables. The tuning procedure uses genetic and neural optimization technique combination. Their model can be applied to commercial programs making prediction of the football games results in bookmaker's offices.

Farzin et al. [1] worked on Football Result Prediction with Bayesian Network in Spanish League-Barcelona Team. They proposed a Bayesian Network (BN) that predicts results of a football match. In their research, they looked at the performance of a Bayesian Network in the area of predicting the result of football matches involving Barcelona FC. The period under study was between 2008-2009 season in Spanish football league and the performance was tested. The required information was obtained from a valid website. Their research shows that $\mathrm{BN}$ is used for prediction of football results in future matches.

Amadin and Obi [3] worked on English Premier League (EPL) Soccer Matches Prediction Using an Adaptive NeuroFuzzy Inference System (ANFIS). Their research uses seven premier league teams and nine linguistic values. The implementation was done using Matlab 7.0. The ANFIS training was successful completed at epoch 2 and having an error of $1.41237 \mathrm{e}-006$. The model was further used to predict the outcome of 7 matches with a successful rate of $71 \%$.

Maral et al. [4] worked on Data Mining Techniques for Result Prediction in Sports. Sports data mining has been discovered to help coaches and managers anticipate results, assess player performance, predict player injury, identify sports talent, and evaluate game strategy. Their study examines prior research on data mining methods for sports prediction and weighs the benefits and drawbacks of each approach.

Promise Enyindah, University of Port Harcourt, Rivers State, Nigeria.

(e-mail: promise.enyindah ${ }^{\circledR}$ uniport.edu.ng) 
Biao Xu [5] worked on Prediction of Sports Performance based on Genetic Algorithm and Artificial Neural Network. He investigated the recent algorithms and proposed a hybrid prediction system based on genetic algorithm and artificial neural network (GANN) in order to predict sports performance more accurately. A database of 1000samples by questionnaire and physical test was created. The dataset was divided into two categories of training set (30\%) and test set $(70 \%)$. The experiments demonstrate the MSE of our method achieves as small as26.637 on the training set and 32.334 on the test set. Moreover, the training time only costs $1.563 \mathrm{~s}$.

Rotshtein et al. [6], worked on Football Predictions based on a Fuzzy model with Genetic and Neural Tuning. They proposed a model for predicting the result of a football match from the previous results of both teams. This model underpins the fuzzy knowledge base method for detecting nonlinear dependencies. By using tournament data to tune fuzzy rules, acceptable simulation outcomes can be attained. The tuning approach entails using a combination of genetic and neural optimization techniques to select the parameters of fuzzyterm membership functions and rule weights.

Tsakonas et al. [7] worked on Forecasting Football Match Outcomes with Support Vector Machines. Their research demonstrates the application of modern statistical or entropybased approaches, such as Support Vector Machines. The results presented in the paper, are indicative of the usability of the SVMs, denoting the competitiveness of this approach among other intelligent approaches for data driven forecasting and decision making.

Omomule et al. [10] a Fuzzy Logic-based prediction model for football matches in their paper. The results of football matches between two teams are generated in their system based on the providence of numerous parameters related with each football team. For each input parameter, the Fuzzy Logic Gaussian Membership function (Gmf) approach is used to compute the membership grade. Datasets were acquired from the twenty (20) English Premier League football teams throughout the 2017/2018 season and used to test the system's predictability. MATLAB version R2017 was used to create their model. When compared to published literature, the evaluation findings demonstrated the system's applicability to the prediction of football outcomes based on the consideration of various input characteristics for improved prediction accuracy.

Onwuachu and Enyindah [12] worked on machine learning application for football player selection. Their proposed model divides the attributes needed for a player selection into four major categories: technique, speed, physical status, and resistance, and uses a neural network technique to determine these major attributes for each player. Matlab 2008 was used to design and implement the system. The results demonstrate that using a neural network to choose players for a football team is a solid idea.

Tsakonas et al. [11] worked on fuzzy rules, neural networks, and genetic programming approaches to develop soft computing algorithms for predicting the outcome of football games. The models consider the following characteristics of football teams: differences in infirmity factors, differences in dynamics profiles, differences in ranks, host factor, and team personal score.
The models achieve a satisfactory estimation of the actual game results, according to testing.

\section{MATERIALS AND METHOD}

Neuro-fuzzy logictechnique was used to develop a model for predicting the outcome of a footballmatch. In the model, differentfactors that affect the result of a football match are analyzed into five major categoriesusing a neural network model. These major categories include the team strategy, the tactical skills, the physical ability, the psychological effect, and the current status. The data used in this paper was collected from this link www.pesstatsdatabase.com/PSD/playerClassic.php?id=1579 $8 \& \mathrm{club}=0$. These major categories will be used in the second phases with the application of fuzzy logic model to generate the optimal prediction results for a football match. The final decision on the football result will be made using fuzzy logic if-then-rules. Fig. 1, Fig. 2, Fig. 3. Fig. 4 and Fig. 5 below shows the neural network model architecture for calculating the team strategy, the tactical skills, the physical ability, the psychological effect, and the current status respectively.

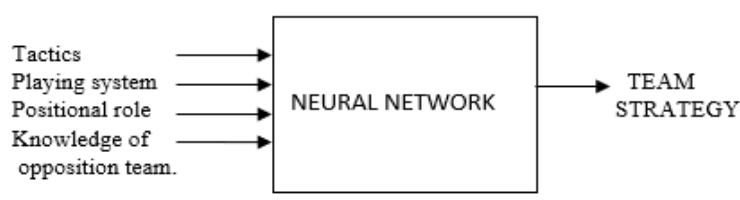

Fig. 1. Neural Network Model Architecture for Team Strategy.

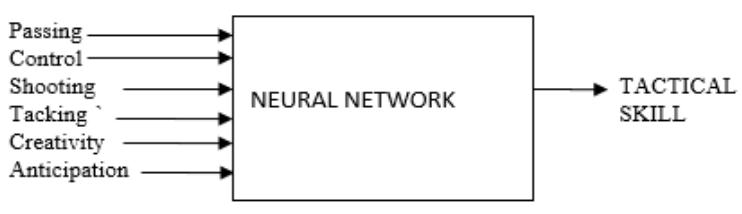

Fig. 2. Neural Network Model Architecture for Tactical Skill.

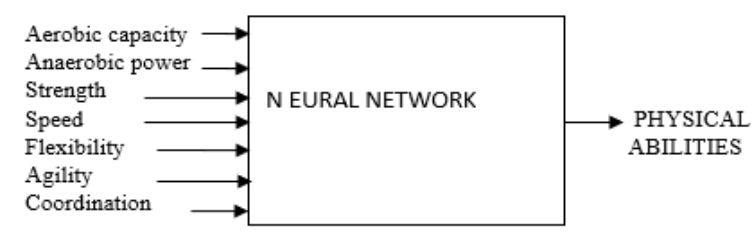

Fig. 3. Neural Network Model Architecture for Physical Abilities.

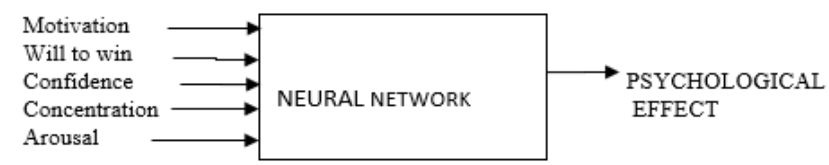

Fig. 4. Neural Network Model Architecture for Phycological Effect.

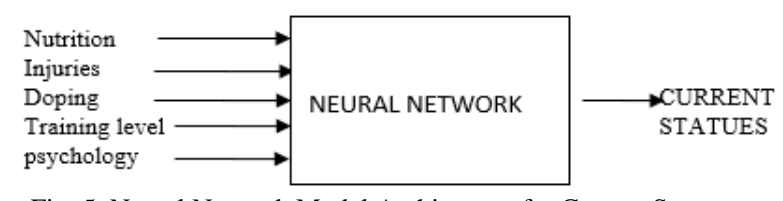

Fig. 5. Neural Network Model Architecture for Current Statues.

The feed forward algorithm was used to calculate theoptimal weights of the individual factors that make upthese categories. The mathematical models for the feedforward algorithm are as follows:

Input $=x_{j}=\sum y_{i} w_{i j}$ 
where $\mathrm{y}_{\mathrm{i}}$ is the generated output and wij represents weights.

$f(x)=1 /\left(1+e^{-x}{ }_{j}\right)$

where $f(x)$ is a sigmoid that is used as the activation function.

Error $=T_{k}-O_{k}$

where $\mathrm{Tk}$ is the observed (True) output while $\mathrm{Ok}$ is the calculated(actual) output.

The error in the output layer is calculated by using the formula in (4):

$\delta \mathrm{k}=\operatorname{Err} k=\mathrm{O} k\left(1-O_{k}\right)\left(T k-O_{k}\right)$

where Ok is the calculated (actual) output expressed in (5):

$O \mathrm{k}=1 /\left(1+\mathrm{e}^{-\mathrm{x}_{\mathrm{j}}}\right)$

where Tk is the observed (True) output.

The back propagation error in the hidden layer is calculated by using the formula in (6):

$\delta \mathrm{j}=\operatorname{Err} j=\mathrm{O} j(1-O j) \sum \delta k * W_{j k}$ where $W_{j k}$ is the weight of the connection from unit $\mathrm{j}$ to unit $\mathrm{k}$ in the next layer and $\delta \mathrm{k}$ is the error of unit $\mathrm{k}$.

The weight adjustment formula in (7) is used to adjust the weights to produce new weights which are feedback into the input layer.

Wnew $=$ Wold $+\eta * \delta *$ input

where $\eta$ is a constant called the learning rate. The learning rate takes value between 0 and 1 .

\section{A. The Basic Structure of a Fuzzy System}

The basic framework of a fuzzy expert system is shown in Fig. 6. Fuzzification module, fuzzy inference engine, fuzzy rule-base, and defuzzification module are the four main modules. Traditionally, the fuzzification module is in charge of taking crisp numeric measurements from the environment, processing them, and mapping them into fuzzy membership function values. The fuzzy engine is in charge of calculating all membership function values using fuzzy sets and communicating with the fuzzy rule base to determine the best fuzzy output. The defuzzification module, on the other hand, is in charge of transforming the fuzzy output into a quantitative output suited for environmental decision-making and control.

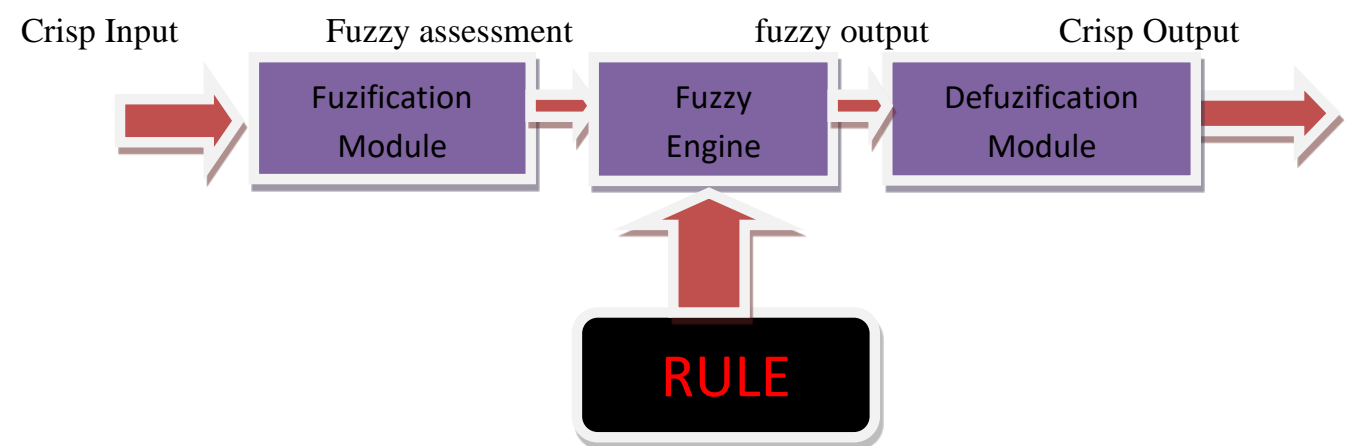

Fig. 6. Basic Architecture of a Fuzzy logic System.

Phase 1

Neural Network model

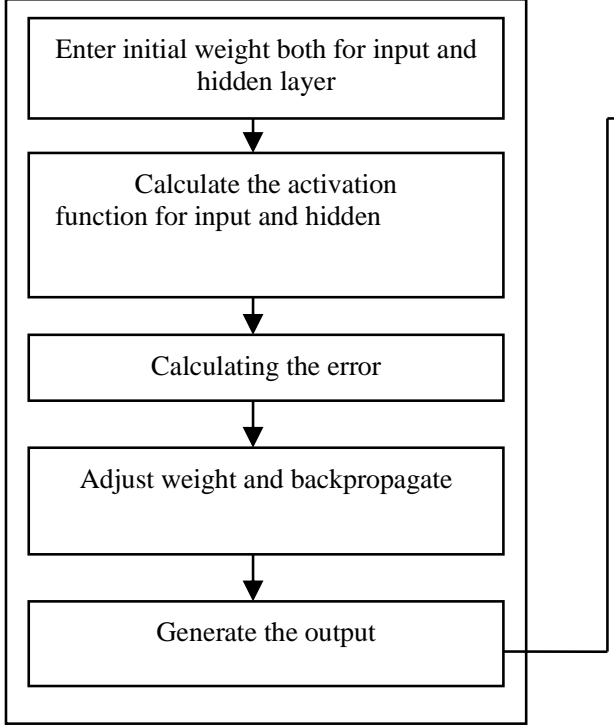

Phase2

Fuzzy Logic model

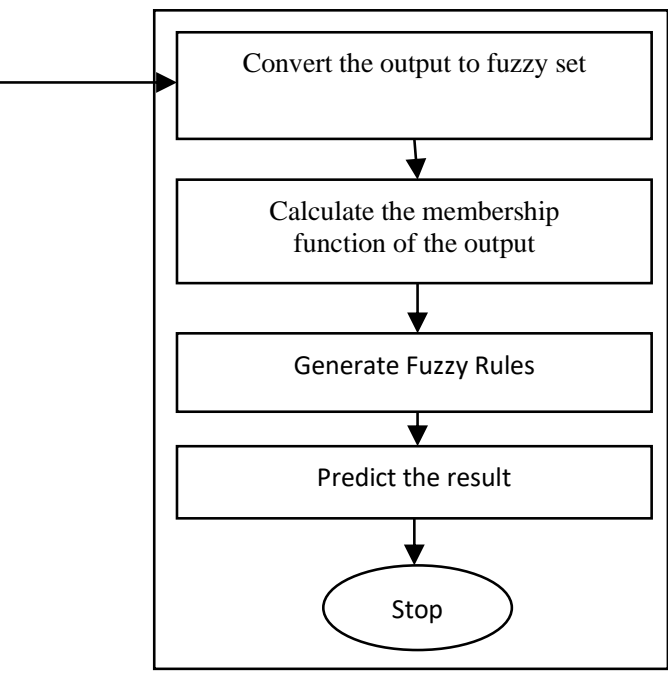

Fig. 7. A diagrammatic representation of the proposed systems framework. 


\section{EXPERIMENTS AND RESULTS}

Fig. 8 shows the Neural Network training. The Neural Network model was trained using Levenberg-Marquardt back propagation. The network is trained to fit the inputs and the target. This means that neural network map between a data of numeric inputs and a set of numeric targets. Training automatically stops when generalization stops improving as indicated by the increase in the mean square error of the validation samples.

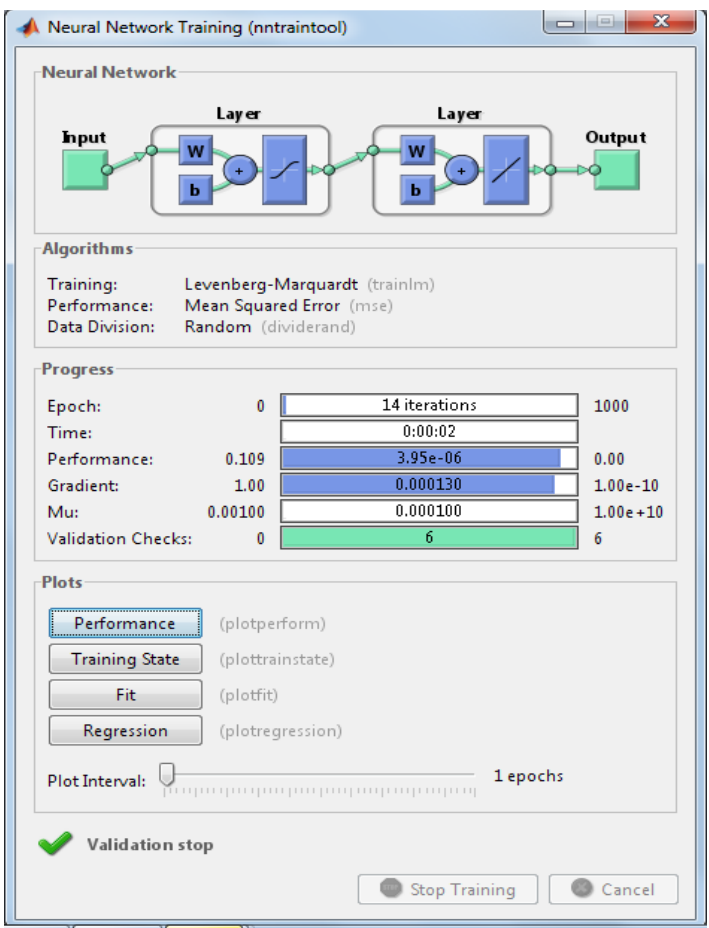

Fig. 8. Neural Network Training Tool.

The neural network fitting tool will help in training network and evaluation its performance using mean square error and regression analysis. Training multiple times will generate different results due to different initial condition and sampling. Fig. 9 shows the result of the trained Network.

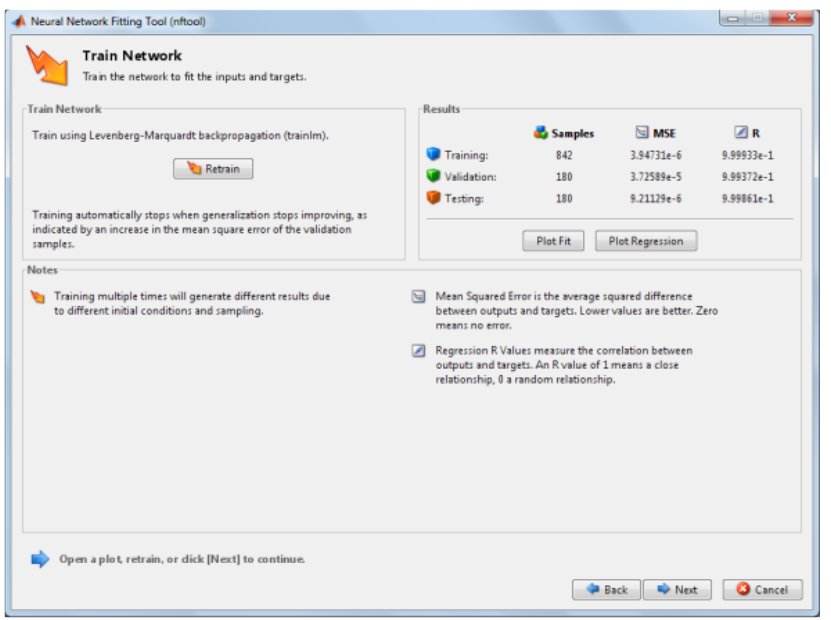

Fig. 9. Neural Network Fitting Tool for Displaying the Result of the Trained Network.

The Membership Function Editor is a tool that allows you to view and edit all of the membership functions associated with all of the fuzzy inference system's input and output variables. As seen in Fig. 10, the Membership Function Editor and the FIS Editor share several functionalities. In reality, the menu selections, status lines, and Help and Close buttons are exactly the same across all basic GUI tools. Fig 10 shows how the membership function for the team's status is displayed.

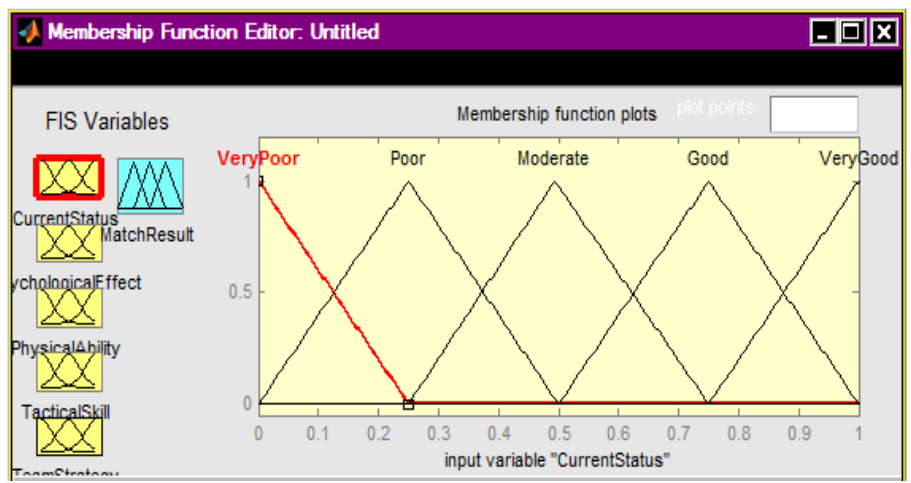

Fig.10. The fuzzy inference membership function editor for team current status.

Constructing rules using the graphical Rule Editor interface is fairly self-evident. Based on the descriptions of the input and output variables defined with the FIS Editor, the Rule Editor allows you to construct the rule statements automatically from the GUI. Fig.11 shows the fuzzy inference rule editor for match prediction.

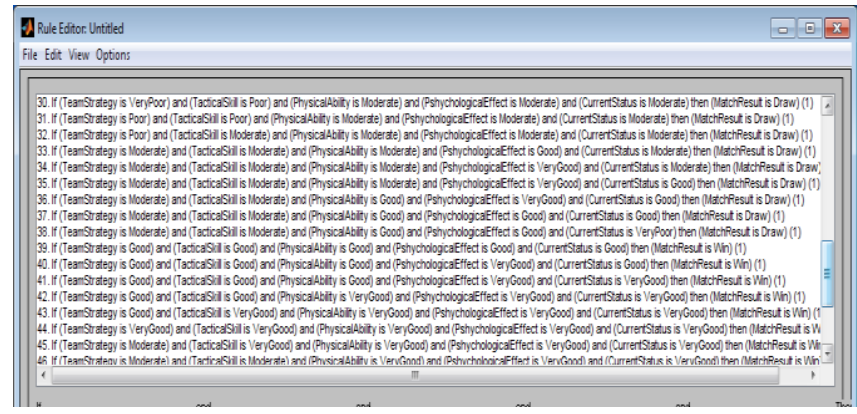

Fig.11.The fuzzy inference system rule editor for match prediction.

The Rule Viewer provides a visual representation of the entire fuzzy inference process. You can use the Rule Viewer to interpret the complete fuzzy inference process at once. It demonstrates how the form of some membership functions has an impact on the final output. The Rule Viewer displays only one computation at a time, and it does so in great detail. It provides a micro perspective of the fuzzy inference system in this way. The fuzzy inference rule viewer for match prediction is shown in Fig. 12.

The three-dimensional curve in the Surface Viewer displays the mapping from team strategy and tactical competence to match result. The complete mapping can be seen in one display because this curve represents a two-input one-output instance. As a result, the Surface Viewer includes drop-down menus $\mathrm{X}$ (input), $\mathrm{Y}$ (input), and $\mathrm{Z}$ output): that allow you to plot any two inputs and one output. Below these menus are two input areas, $\mathrm{X}$ grids: and $\mathrm{Y}$ grids, which allow you to specify the number of $\mathrm{x}$ - and $\mathrm{y}$-axis grid lines you want to include. The fuzzy inference surface viewer for match prediction is shown in Fig. 13. 


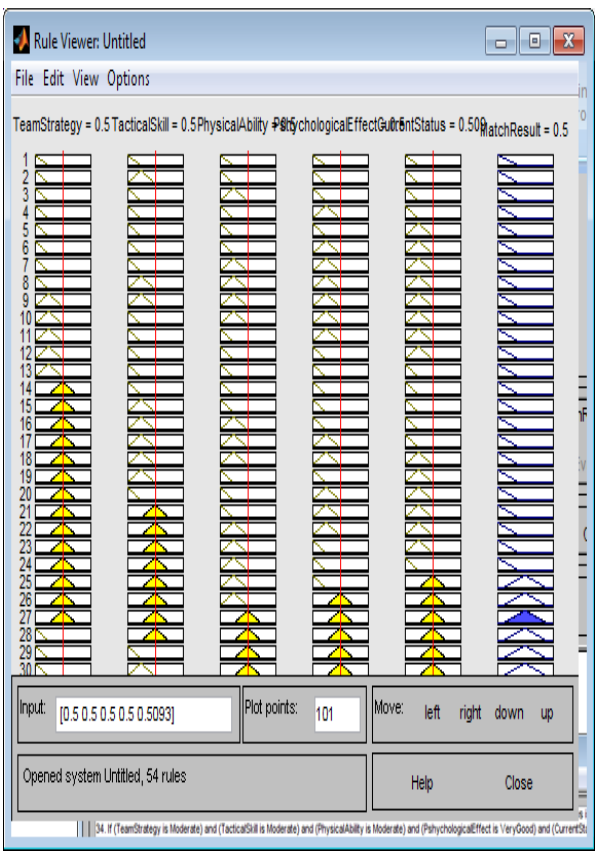

Fig.12.The fuzzy inference rule viewer for match prediction.

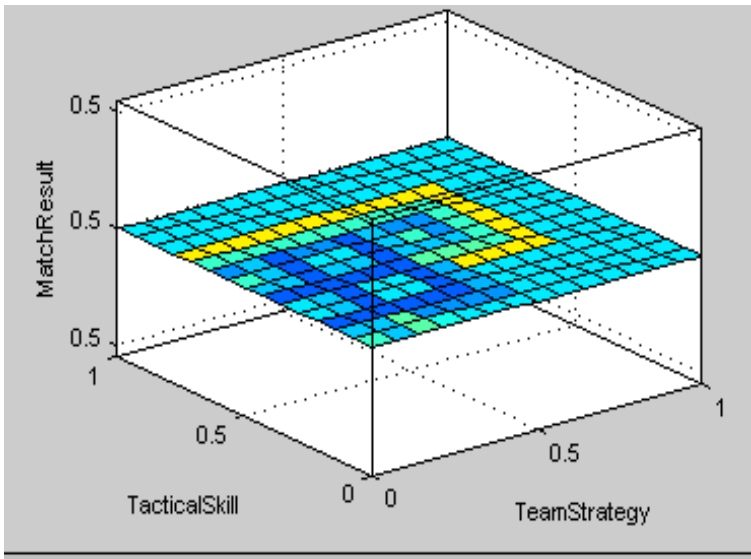

Fig.13.The fuzzy inference surface viewer for match prediction.

\section{RESULT DISCUSSION}

Fig. 8shows the Neural Network training. LevenbergMarquardt back propagation was used to train the Neural Network model. The network is honed to match the inputs and outputs. Training comes to an end automatically when generalization stops rising, as evidenced by a rise in the mean square error of the validation samples. Using mean square error and regression analysis, the neural network fitting tool will aid in network training and performance evaluation. Training many times will produce different outcomes due to shifting beginning circumstances and sampling. The trained Network's outcome is shown in Fig. 9. During this step, the neural network's output is utilized. The Membership Function Editor and the FIS Editor share various functions, as seen in Fig. 10. The membership function for the present status of the team is displayed in the figure. It's quite self-evident how to create rules using the graphical Rule Editor interface. The Rule Editor allows you to automatically generate rule statements from the GUI based on the descriptions of the input and output variables defined with the FIS Editor. The fuzzy inference rule editor for match prediction is shown in Fig. 11. The Rule Viewer provides a visual representation of the entire fuzzy inference process. You can use the Rule
Viewer to interpret the complete fuzzy inference process at once. It demonstrates how the form of some membership functions has an impact on the final output. The Rule Viewer displays only one computation at a time, and it does so in great detail. It provides a micro perspective of the fuzzy inference system in this way. The fuzzy inference rule viewer for match prediction is shown in Fig. 12. The threedimensional curve in the Surface Viewer displays the mapping from team strategy and tactical competence to match result. The complete mapping can be seen in one display because this curve represents a two-input one-output instance. As a result, the Surface Viewer includes drop-down menus $\mathrm{X}$ (input), Y (input), and Z output): that allow you to plot any two inputs and one output. Below these menus are two input areas, $\mathrm{X}$ grids: and $\mathrm{Y}$ grids, which allow you to specify the number of $\mathrm{x}$ - and $\mathrm{y}$-axis grid lines you want to include. The fuzzy inference surface viewer for match prediction is shown in Fig. 13.

\section{CONCLUSION}

The paper demonstrated that a Neurofuzzy logic system can be used to predict the outcome of a football match by taking into account the major factors that influence the outcome of a football match, such as team strategy, tactical skills, physical ability, psychological impact, and current team status. In order to forecast the winner and loser of each football match, the model took into account a variety of parameters that affect both the host team and the visiting squad. The results show that the Neurofuzzy logic technique is an effective tool for forecasting the outcome of a football match.

\section{REFERENCES}

[1] Farzin Owramipur, Parinaz Eskandarian, and Faezeh Sadat Mozneb Football Result Prediction with Bayesian Network in Spanish LeagueBarcelona Team. International Journal of Computer Theory and Engineering, 2013;5(5).

[2] Alexander Rotshtein, Morton Posner and Hanna Rakytyanska. Prediction of the Results of Football Games Based on Fuzzy Model with Genetic and Neuro Tuning. Eastern European Journal of Enterprise Technologies, 2003:10-18.

[3] Amadin F. I and Obi J.C.English Premier League (EPL) Soccer Matches Prediction Using An Adaptive Neuro-Fuzzy Inference System (ANFIS). Transact ions on Machine Learning and Artificial Intelligence, 2015;3(2).

[4] Maral Haghighat, Hamid Rastegari and Nasim Nourafza. A Review of Data Mining Techniques for Result Prediction in Sports. ACSIJ Advances in Computer Science: An International Journal, 2013;2(5;6).

[5] Biao Xu. Prediction of Sports Performance based on Genetic Algorithm and Artificial Neural Network. International Journal of Digital Content Technology and its Applications (JDCTA), 2012;6(22):14-25.

[6] Rotshtein A. P., PosnerM., and Rakityanskaya A. B. Football Predictions based on a Fuzzy model with Genetic and Neural Tuning. Cybernetics and Systems Analysis, 2005;41(4)

[7] Tsakonas A., Dounias G., and Shtovba S. Forecasting Football Match Outcomes with Support Vector Machines. Herald of Zhytomyr Engineering - Technological Institute, 2003;1:181-186

[8] Miljkovic D., Gajic L., Kovacevic A., Konjovic Z. The use of data mining for basketball matches out comes prediction. IEEE 8th International Symposium on intelligent and informatics, Subotica, Serbia, 2010, pp. 309-312.

[9] Yudong Zhang, Shuihua Wang, Lenan Wu, Yuankai Huo. PSONN used for Remote-Sensing Image Classification. Journal of Computational Information Systems, 2010;6(13):4417-442. 
[10] Omomule T.G., Ibinuolapo A. J, Ajayi O.O. Fuzzy-Based Model for Predicting Football Match Results. International Journal of Scientific Research, Dept. of Computer Science, Adekunle Ajasin University, Akungba Akoko, Ondo, 2020;S8(1):70-80.

[11] Tsakonas A., Dounias G., Shtovba S., Vivdyuk V. Soft ComputingBased Result Prediction of Football Games. The First International Conference on Inductive Modelling, 2002;3(1):15-21.

[12] Onwuachu Uzochukwu C. and P. Enyinda. A Machine Learning Application for Football Players' Selection. International Journal of Engineering Research \& Technology (IJERT), 2015;4(10):459-465. 